\title{
MODERN METHODS OF MONITORING VARIABLES FOR RACEHORSES
}

\author{
Veronika Hartova, Jan Hart \\ Czech University of Life Sciences Prague \\ nidlova@tf.czu.cz
}

\begin{abstract}
Currently there is a big trend to watch different variables for racing horses. There is great interest to monitor their condition in the race. This monitoring leads to the optimization of the needs of race horeses, which improves their final outputs. It is important to many countless indicators such as temperature, humidity, pressure, heart rate and many other to be monitored. It is therefore necessary to define a simple system for transferring the recorded data. This system must be reliable, flexible and especially efficient. Due to the the need for fast data transfer this technology must be wireless. The basis therefore is to create a so-called "multiple- sensor network". This network must allow for variability and allow mesh communication. The proposed network must then allow forwarding of messages, because each transmitter is also the receiver (repeater function). A survey was conducted on the current applicable technology. Eventually, the IQRF technology has been selected that exactly matches the specified criteria. The IQRF technology operates at a frequency of $868 \mathrm{MHz}$, which corresponds to the ISM868 band (Industrial, Scientific and Medical). IQRF mesh allows communication and is suitable for distances of tens to hundreds of meters. Its dimensions are small (about $2 \mathrm{~cm}$ long, $1 \mathrm{~cm}$ wide and $0.25 \mathrm{~cm} \mathrm{high),}$ allowing seamless integration. It has been found that it is necessary to place the track stationary points. However, it is not necessary to solve the remote transmitter that would send its information which is identified from these stationary points. Because it is mesh communication, each loop element communicates with each other and does not require other transmit interfaces.
\end{abstract}

Keywords: racehorse, horse, monitoring, IQRF, wireless transmissions, sensor network.

\section{Introduction}

One of the main goals of the research on monitoring race horses is the area of simple data transfer. This always requires investigation supported by measurements, which consist of the process of evaluation and gaining of the needed data [1].

These measurements are used to evaluate the external conditions, which affect the racehorses both directly and indirectly.

Currently, the most widespread are portable measuring instruments that are used for discontinuous measurement, or long-term measuring instruments located at points of interest for the long term (offline sensor at the racehorse).

This mainly involves (offline) system sensor networks without simple wireless transmissions or (online) system sensor networks with simple wireless transmissions [2].

Of course, there are also many risks and imperfections related to wireless networks that need to be considered. One of the most frequent risks of wireless transmission is natural interference or a limited range of wireless transmitters. Though being serious, these problems have their realistic solutions provided by an IQRF mesh topology network, which can at least partially eliminate them. The IQRF transmission parameters are as follows [2;3]:

- sonsumption: 8.3-19 mA;

- transmission speed: $19.836 \mathrm{~kb} \cdot \mathrm{s}^{-1}$;

- packet data: up to 64 bytes of user data;

- transmission time: $30-50 \mathrm{~ms}$ per node.

\section{Materials and methods}

To thoroughly understand the problems, it is needed to define the type of data paths to be subsequently compared. Because of monitoring the development of the situation in the field only continuous forms of monitoring will be evaluated and, in turn, the data paths, which can be used for this monitoring. Basic types of data transmission include a classic wireless transmission with repeaters and mesh type wireless transmission. "Classic" wireless networks with repeaters use star topology as counterpart to mesh topology.

A classic wireless transmission using repeaters (see Fig. 1) will operate without wired connection with individual sensors, which in part reduces the problems relating to cable laying as well as final 
removing of the sensor network. Although it is used quite frequently, it includes a risk of loss of critical points (repeaters) and possibly even loss of data due to natural interference, which may impact the data paths. In this case it involves the communication between specific network nodes (detector main unit) [4].

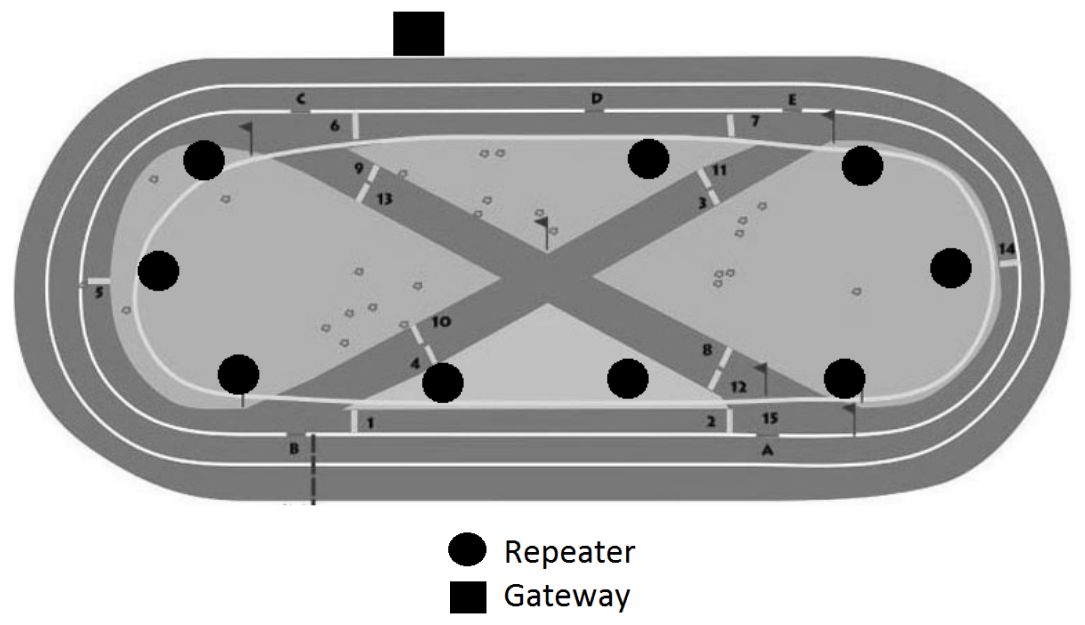

Fig. 1. Wireless network distribution through stationary repeaters on racecourse

Regarding the classic wireless network we must not forget that each monitored entity has a transmitter on it, which transfers information through repeaters to the gateway - see Fig. 2. It is essential in this connection that repeaters must cover the whole monitored area and the way the data are always precisely determined [5].

Mesh type wireless transmission topology is a relatively new method, which could find an extensive use right in the measurement of racehorses. In this kind of transmission each transmitter also works as a repeater. This significantly reduces the loss of the needed information as the network points are mutually interchangeable. Therefore, the failure of one network element does not create a situation where all communication would be disrupted, but only the one affected specific element of the sensor network will drop out. The image would look like in the previous case. But there is a fundamental change. Repeaters are classically wireless transmission different from conventional transmitters. They are usually expensive and mostly with the necessity of an external power supply. In mesh networks each particular node is also a repeater [5; 6].

At first sight, the classic wireless transmission through a mesh network is almost interchangeable. Both options require stationary repeaters that send the information from the transmitters to the gateway. However, taking a closer look reveals that individual transmitters send their transferred data to all other transmitters within their reach and these repeat their message - see Fig. 3. This enhances the network security and reduces the number of needed repeaters as compared to the classic wireless network. In this type of transmission repeaters do not need to cover the whole monitored space.

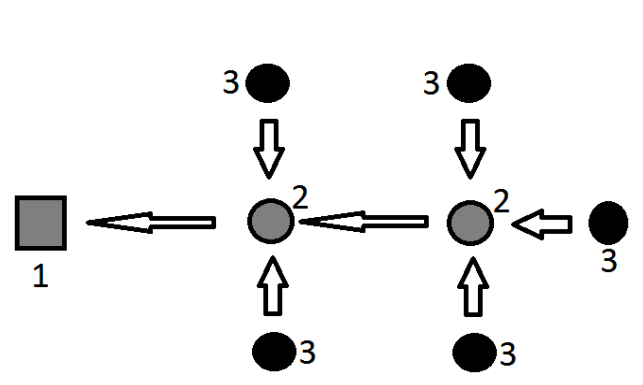

Fig. 2. Transfer principle for classic wireless network: 1 - gateway; 2 - repeater; 3 wireless sensor

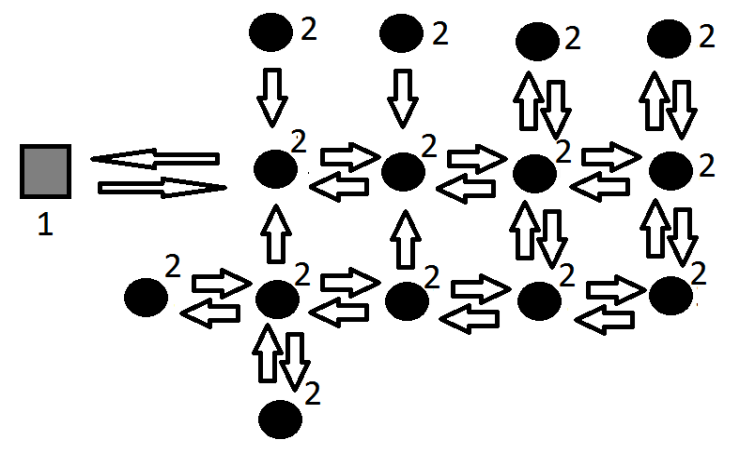

Fig. 3. Transfer principle for mesh wireless network: 1 - gateway; 2 - node 
Advantages and disadvantages of a single method for wireless data have been compared through a percentage of multi-criteria analysis of options with an assigned weighting coefficient. It is true that the lower the weighting coefficient, the more important the criterion is being evaluated. They were selected following the evaluation criteria, which have been selected:

- price of repeaters (weighting coefficient: 4);

- need to use disparate technologies (weighting coefficient: 4);

- security of transmission lines (weighting coefficient: 1 );

- ease of installation (weighting coefficient: 3 );

- substitutability of individual elements (weighting coefficient: 3 );

- communication method (weighting coefficient: 2);

- frequency variation (weighting coefficient: 2);

- power efficiency (weighting coefficient: 1 );

- transfer speed (weighting coefficient: 1 ).

Weighting coefficients were determined on the basis of recommendations from several industry experts. For comparison the following systems were selected:

- IQRF (mesh);

- Bosch (mesh);

- Jablotron (AC-82 with accessories);

- Siemens (Synco).

\section{Results and discussion}

Weight values for the percentage of multi-criteria analysis of options with an assigned weighting coefficient where this method was modified for the individual types of connection in the way that they were assigned, a score for each item on a 100-point scale is based on the set parameters. The resulting scores for both methods were then converted to percents and shown in Figure 4.

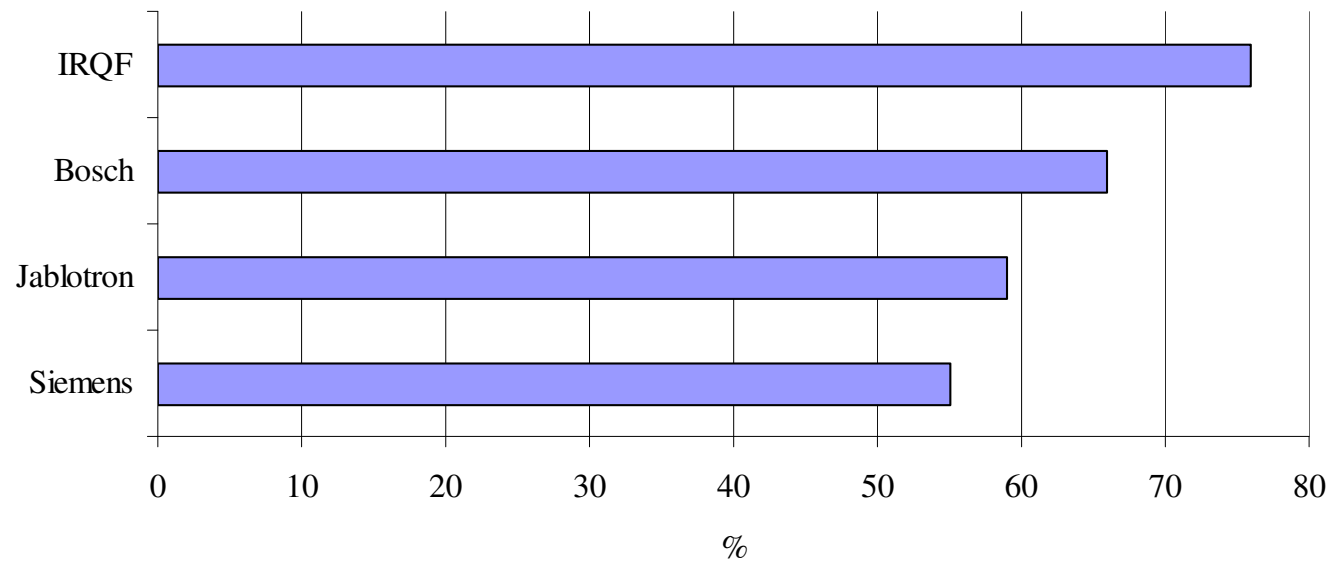

Fig. 4. Results of multi criteria analysis of the options

It is obvious that systems of the type mesh are based on comparing best. It has therefore been created, yet mesh was compared with the classic networks, which we see in Figure 5 below.

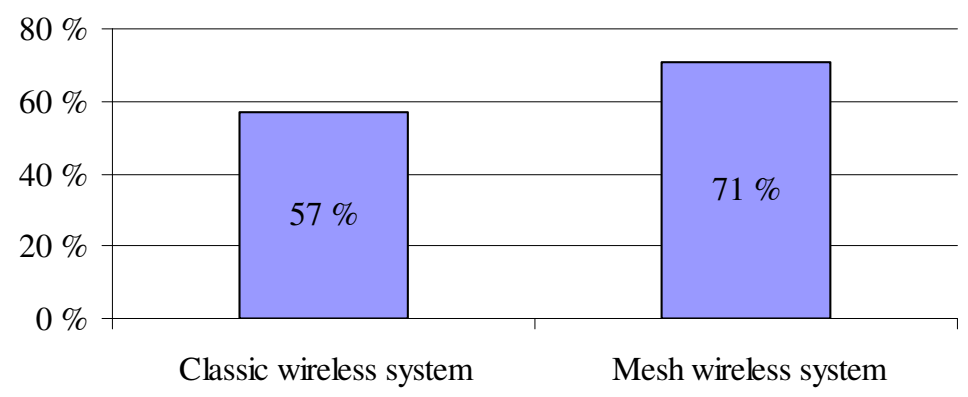

Fig. 5. Comparison of classical wireless network with network of type mesh 
Based on the comparison of these technologies the IQRF system was chosen for the design of the transmitter. The transmitter is integrated into the saddle connection for racehorses. It was also needed to devise a hardware connection of the transmitter to possibility connect it with the various sensors located beneath the riding saddle.

The hardware design primarily considered a functional and minimalist solution. There were node inputs and outputs defined and a basic printed circuit design was made for their integration - see Fig. 6. Next specific fitting of the printed circuit was designed so as to correspond with the final purpose - see Fig. 7.

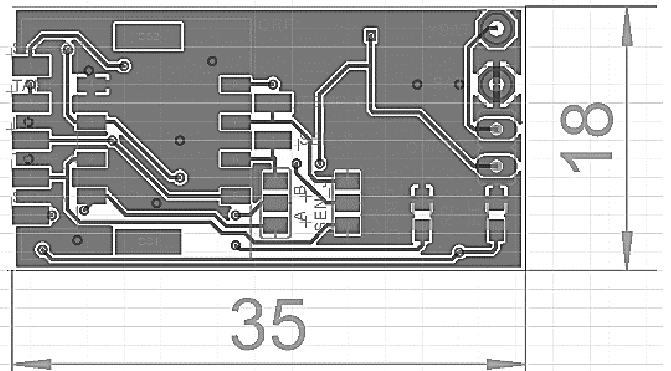

Fig. 6. Designed size and layout of printed circuit

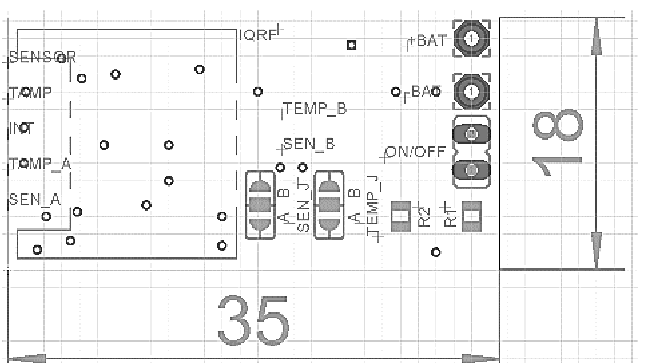

Fig. 7. Design of printed circuit

Finally, an exact description of individual connections of the nodes to the hardware platform was created (Fig. 8), so that they can replace the sensors on the racehorses and convert transfer to wireless communication.
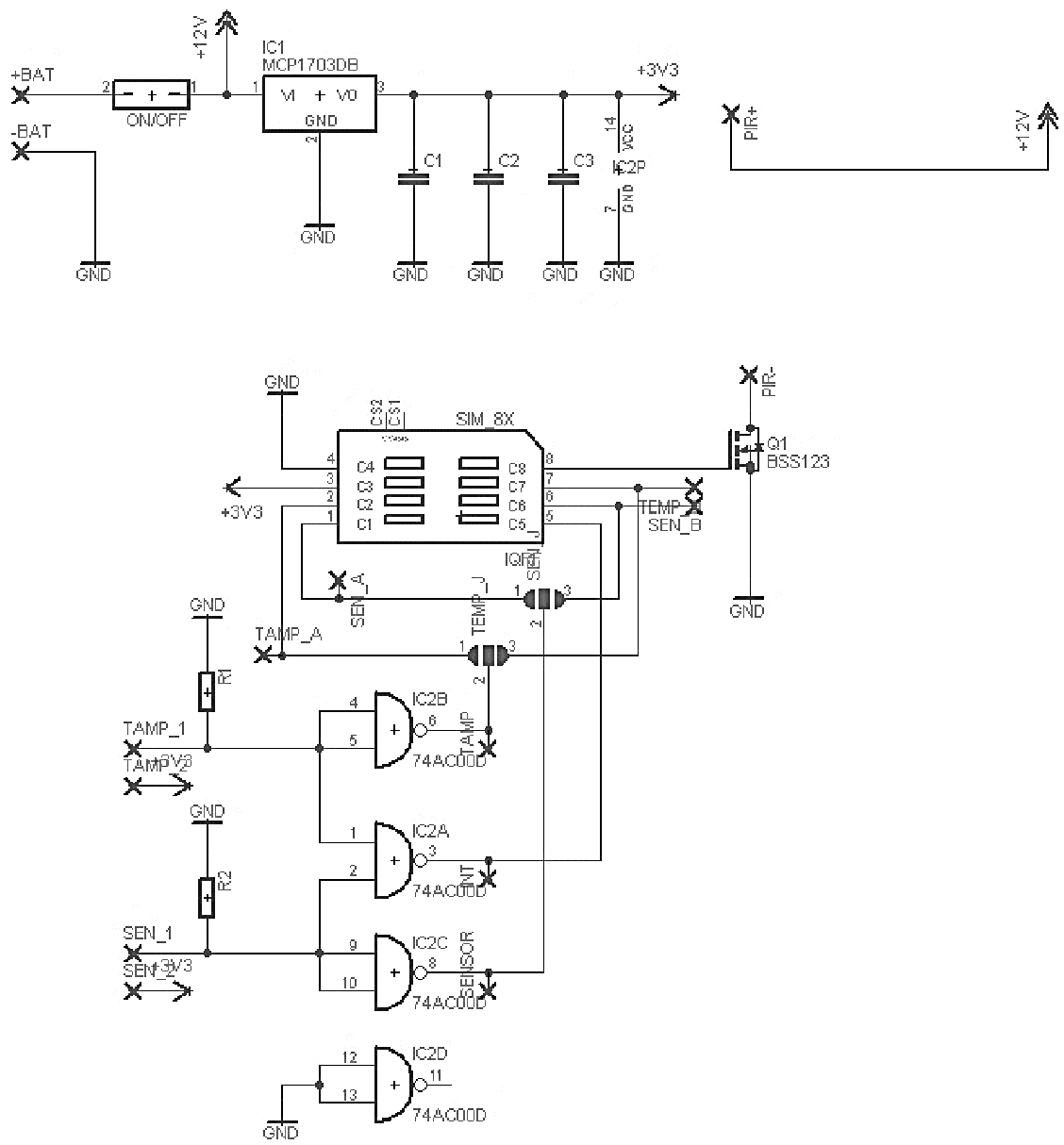

Fig. 8. Node connection to hardware platform 
The above figure shows the IQRF Wireless Charge Interface for Sensor Interfacing. The sensor input is marked as SENSOR. The tamper input is marked as TAMP. Tamper monitors whether the measuring box is closed.

Next, the nodes were set up so that one was placed as stationary at the exchange point (concentrator) and the other one was put right in the detector. After that the concentrator was connected to the ethernet gateway and to the exchange entry so as to be able to transmit the sensor information.

As described by the authors in the articles on "Building IoT Applications with Raspberry Pi and Low Power IQRF Communication Modules" [7], "Use of IQRF Technology for Detection of Construction Inclination" [3] and "Wireless Temperature Measurement System Based on the IQRF Platform" [1], IQRF is a variable and multifunctional platform providing a broad range of use.

A similar view is taken by the authors of the articles "IQRF Wireless Technology Utilizing IQMESH Protocol" [4], "IQRF Smart Wireless Platform for Home Automation: A Case Study" [2], and "IQMESH implementation in IQRF wireless communication platform" [5], though they express some scepticism about the local character of using this technology.

\section{Conclusions}

Based on this comparison the printed circuit design was subsequently developed for integrating an IQRF node for transmission of basic temperature data in order to monitor racehorses while considering the monitoring of individual animals. More information can be derived from the monitoring of individual racehorses than from mass monitoring. A greater amount of variables are recorded that can be monitored and evaluated, and decisions can be taken based on them as to which way to choose for moving forward and how to modify the present conditions.

Natural interference, which may affect wireless transmission, occurs rather rarely, still it is important for wireless transmissions to be able to either identify such interference or replace the path of transfer. Mesh systems do possess this feature and therefore are more secure in real life operation than standard wireless systems.

The resulting design has its selected hardware construction and it has clearly proven through the node programming that IQRF fully supports the IoT logic and it satisfies its exact definition which reads as follows: "The resulting device should be wireless in the first place, and it should bring new possibilities of mutual interaction not only between the individual systems, but also on a global scale. The device should also provide new options of their control, monitoring and provision of advanced services." Thanks to the created unified modifiable system it was demonstrated as well that it enables easy updating for a different use than that it was originally set up for and thus becomes a multifunctional system following such update.

Although IQRF appears to be a perfect tool for IoT implementation, it is primarily a local adaptation of wireless transmissions. As long as the manufacturer has not commenced full cooperation with the other variants of wireless transmission over a larger distance, such as Sigfox and Lora, the system's use will remain limited, even when meeting the condition of being a technology, which fully conforms to IoT demands.

\section{References}

1. Bazydlo P., Dabrowski S., Szewczyk R. Wireless Temperature Measurement System Based on the IQRF Platform, In: International Conference on Mechatronics - Ideas for Industrial Applications, Lodz, Poland, Volume: 317, 2015, pp. 281-288.

2. Kuchta R., Vrba R. Sulc V. IQRF Smart Wireless Platform for Home Automation: A Case Study, In: 5th International Conference on Wireless and Mobile Communications, Cannes, France, 2009, pp. 168-173.

3. Martin P., Radovan, H. Use of IQRF Technology for Detection of Construction Inclination, In: International Conference on Numerical Analysis and Applied Mathematics (ICNAAM), Volume: 1738, 2016, Article Number: 480121. 
4. Seflova P., Sulc V., Pos J., Spinar R. IQRF Wireless Technology Utilizing IQMESH Protocol, In: 35th International Conference on Telecommunications and Signal Processing (TSP), Prague, Czech Republic, 2012, pp. 101-104.

5. Sulc V., Kuchta R., Vrba R. IQMESH implementation in IQRF wireless communication platform, In: 2nd Interational Conference on Advances in Mesh Networks, Athens, Greece, 2009, pp. 62-65.

6. Tahir H., Shah S.A.A. Wireless Sensor Networks - A Security Perspective, In: International multitopic conference, 2008, pp. 189-193.

7. Calvo I., Gil-Garcia J.M., Recio I., Lopez A., Quesada J. Building IoT Applications with Raspberry Pi and Low Power IQRF Communication Modules, Electronics, Volume: 5, Issue: 3, 2016, Article Number: 54. 